\title{
Expressive Power of Safe First-Order Logical Decision Trees
}

\author{
Joris J.M. Gillis` and Jan Van den Bussche \\ Hasselt University and transnational University of Limburg
}

\begin{abstract}
This paper characterizes the expressive power of a subclass of first-order logical decision trees (FOLDTs) as a fragment of firstorder logic. Specifically, using safe FOLDTs one can express precisely the boolean combinations of safe existential sentences.
\end{abstract}

\section{Introduction}

In Logical and Relational Learning [5], the logical languages that can be learned most effectively offer rather limited expressiveness, typically not going beyond the existential fragment of first-order logic. Indeed, this is the standard balancing exercise between expressive power and efficiency that one faces everywhere in the fields of AI and computer science. First-order logical decision trees (FOLDTs) [1] are one of the few logical languages used in ILP that offer higher expressive power, yet can still be learned effectively (cf. the Tilde system, part of the ACE-ilProlog system $[2,3]$ ). FOLDTs allow the expression of certain properties involving universal quantification in a natural and direct manner. For example, consider the vocabulary with a binary relation symbol $E$, used to indicate the edges of a directed graph, and unary relation symbols $R$ and $B$, used to indicate the "red" and the "blue" nodes in the graph. Then the very simple FOLDT shown in Fig. 1 expresses the property that every blue node has edges to all red nodes, expressed in first-order logic as

$$
(\forall x)(B(x) \rightarrow(\forall y)(R(y) \rightarrow E(x, y))) .
$$

A natural question now, which has remained unanswered in the literature so far, is, exactly which properties can be expressed by FOLDTs? Blockeel and De Raedt [1] have given a translation of FOLDTs into recursion-free Prolog which can be equivalently expressed in first-order logic (FOL). However, exactly which fragment of FOL do we cover by FOLDTs? In the present paper we answer the question for a subclass of FOLDTs, called the safe FOLDTs and show the equivalence between safe FOLDTs and the fragment of FOL formed by all safe boolean combinations of existential sentences. For example, the above formula can be rewritten as the negation of an existential sentence:

$$
\neg(\exists x, y)(B(x) \wedge R(y) \wedge \neg E(x, y))
$$

\footnotetext{
* Ph.D. Fellow of the Research Foundation Flanders (FWO).
} 


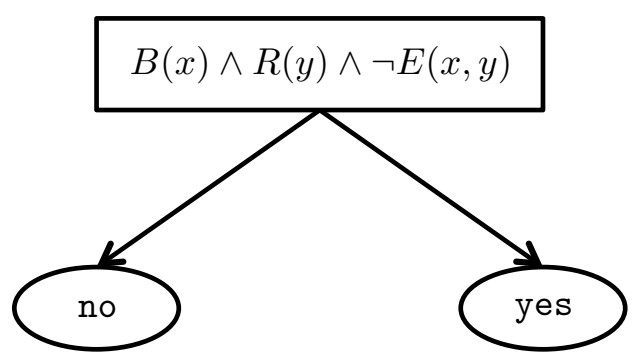

Fig. 1. Example of a FOLDT.

This existential sentence is safe because variables $x$ and $y$, which occur in the negative literal $\neg E(x, y)$, also occur in a positive literal $(B(x)$ and $R(y)$ respectively). A formal definition of safety will be provided later in the paper.

Note that formula $(\dagger)$ closely matches the FOLDT of Fig. 1.

Our result implies that properties whose expression require the alternation of quantifiers in an essential way are not expressible as a safe FOLDT. A typical example of such a property is "there exists a blue node with edges to all red nodes", or in FOL,

$$
(\exists x)(B(x) \wedge(\forall y)(R(y) \rightarrow E(x, y))) .
$$

It is interesting to put our result in the perspective of the logical learning model by Osherson and Weinstein [7,8,9]; see also [6]. Indeed, there it has been shown that a query is learnable in the limit if and only if it is $\Delta_{2}$-expressible. Since our result places safe FOLDTs in $\Delta_{2}$, as we will see in section 5 , our result may help to explain why FOLDTs are effectively learnable (in the limit).

\section{Preliminaries}

To avoid misunderstanding, we fix terminology and notation for some well-known notions from logic. A relational vocabulary is a set $\tau$ of relation symbols, each with an associated arity (a natural number). A $\tau$-interpretation $I$ consists of a nonempty set $\operatorname{dom}(I)$, called the domain of $I$, and a $k$-ary relation $R^{I}$ on $\operatorname{dom}(I)$, for each $R \in \tau$, with $k$ the arity of $R$.

A boolean query over $\tau$ is a function $Q$ from the set of $\tau$-interpretations to the two-element set $\{$ yes, no $\}$. In the most basic classification setting of learning from interpretations, the learner is provided with some yes- and some no-instances of a boolean query $Q$, and must infer a classifier, i.e., an expression for $Q$. Often this expression can be translated in a first-order logic (FOL) sentence; this is the case, for example, with classifiers in the form of recursion-free Prolog programs. A FOL sentence over $\tau$ is a FOL formula without free variables and involving only the relation symbols from $\tau$, besides the equality symbol. The boolean query $Q$ expressed by such a sentence $\varphi$ is defined as follows: for every $\tau$-interpretation 
$I$, we have that $Q(I)=$ yes if and only if $I \models \varphi$. Here, $I \models \varphi$ denotes that $\varphi$ is true in $I$. The class of queries expressed by FOL formulas is called the class of first-order queries.

\section{First-Order Logical Decision Trees}

Fix a relational vocabulary $\tau$. Recall that a literal is an atomic formula with relation name from $\tau$, or the negation of such an atomic formula. A first-order logical decision tree (FOLDT) over $\tau$ is a couple $(T, \lambda)$, such that

$-T$ is a finite binary tree; and

$-\lambda$ is a labeling function on the nodes of $T$ such that each internal node (including the root) is labeled with a conjunction of literals over $\tau$, and each leaf node is labeled with 'yes' or 'no'; the label of node $n$ is denoted by $\lambda_{n}$.

When no confusion can arise, we will refer to the FOLDT simply as $T$ and leave $\lambda$ implicit.

An example of a FOLDT over the vocabulary consisting of the two binary relation names $R$ and $S$, is shown in Figure 2 .

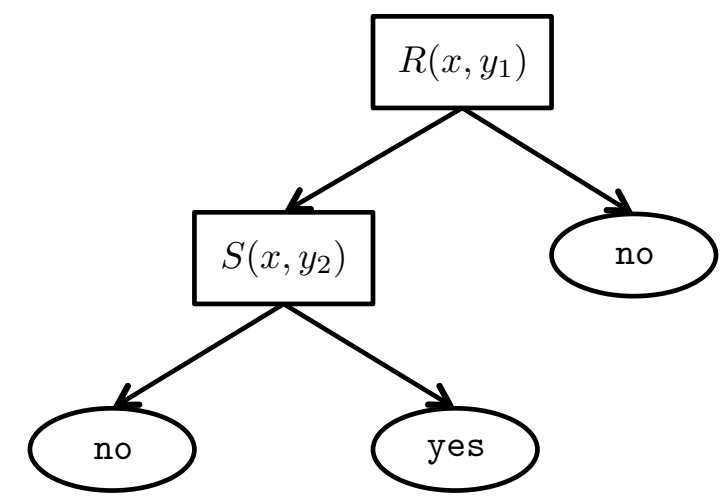

Fig. 2. FOLDT expressing the first-order query $\exists x\left(\exists y_{1} R(x, y) \wedge \neg \exists y_{2} S\left(x, y_{2}\right)\right)$.

The semantics of FOLDTs has been defined by a translation into recursionfree Prolog [1]. The resulting Prolog programs use negation by failure which sometimes gives non-declarative results. Consider, for example, the simple FOLDT consisting of a root with two leaves as children. The left child is labeled yes, the right child is labeled no. The root is labeled $\neg R(x)$. Then the semantics of this FOLDT is equivalent to $\neg(\exists x) R(x)$. In contrast, if the root would be labeled $S(x) \wedge \neg R(x)$, the semantics would become $(\exists x)(S(x) \wedge \neg R(x))$. Moreover, if the root would be labeled $\neg R(x) \wedge S(x)$, the semantics would be $\neg(\exists x)(R(x)) \wedge$ $(\exists x)(S(x))$. 
We next define a more declarative semantics for FOLDTs, which is equivalent to the original Prolog semantics in the case the FOLDTs are safe. We will define safety below, but first we already give the semantics.

A FOLDT $T$ expresses a Boolean first-order query $Q_{T}$. The query $Q_{T}$ is defined by translating $T$ into a FOL sentence $\Phi_{T}$.

1. We first define formulas $\alpha_{n}$ for every node $n$ of $T$ :

$$
\alpha_{n}:= \begin{cases}\text { true } & \text { if } n \text { is the root of } T \\ \alpha_{p} \wedge \lambda_{p} & \text { if } n \text { is the left child of node } p \\ \alpha_{p} \wedge \neg(\exists \bar{y})\left(\alpha_{p} \wedge \lambda_{p}\right) & \text { if } n \text { is the right child of node } p .\end{cases}
$$

where $\bar{y}$ is the set of free variables in $\alpha_{p} \wedge \lambda_{p}$.

2. For each node $n$ we next define $\beta_{n}$ as the formula $(\exists \bar{z})\left(\alpha_{n}\right)$, where $\bar{z}$ is the set of free variables in $\alpha_{n}$.

3. Finally, we define

$$
\Phi_{T}:=\bigvee\left\{\beta_{\ell} \mid \ell \text { is a leaf node labeled yes }\right\} .
$$

Example 1. Consider the FOLDT $T$ of Fig. 2. Let us number the root, the left child of the root, and the only leaf node labeled yes, as 1, 2, and 3, respectively. Then

$$
\begin{aligned}
& \alpha_{1}=\text { true } \\
& \alpha_{2}=\left(\operatorname{true} \wedge R\left(x, y_{1}\right)\right) \equiv R\left(x, y_{1}\right) \\
& \alpha_{3}=R\left(x, y_{1}\right) \wedge \neg\left(\exists x, y_{1}, y_{2}\right)\left(R\left(x, y_{1}\right) \wedge S\left(x, y_{2}\right)\right)
\end{aligned}
$$

and we obtain $\Phi_{T}=\left(\exists x, y_{1}\right)\left(\alpha_{3}\right)$ :

$$
\left(\exists x, y_{1}\right) R\left(x, y_{1}\right) \wedge \neg\left(\exists x, y_{1}, y_{2}\right)\left[R\left(x, y_{1}\right) \wedge S\left(x, y_{2}\right)\right] .
$$

\subsection{Safety}

Let $n$ be a node of a FOLDT $T$. Let $A_{n}=\left(m_{1}, \ldots, m_{k}\right)$ be the sequence of nodes from the root $r=m_{1}$ to node $n\left(m_{k}\right.$ is the parent of $n$ and $m_{k+1}$ denotes $n)$. The leftish ancestors of $n$ are the nodes $m_{i}, 1 \leq i \leq k$, in $A_{n}$ such that $m_{i+1}$ is the left child of $m_{i}$.

Example 2. The fragment of a FOLDT depicted in Fig. 3 has three nodes of interest. The root, node 1, has no leftish ancestors, because it is the root. Node 2 has the root as sole leftish ancestors. The root is the sole leftish ancestor of node 3 . Indeed, $A_{3}=(1,2,3)$, node 2 is a left-child of 1 and thus is 1 a leftish ancestor.

The conjunction $\lambda_{n}$, labeling node $n$, is a sequence of literals. The local predecessors of the occurrence of a literal $\xi$ in $\lambda_{n}$ is the set of literals syntactically preceding $\xi$ in $\lambda_{n}$. The global predecessors of $\xi$ are the literals of the leftish 


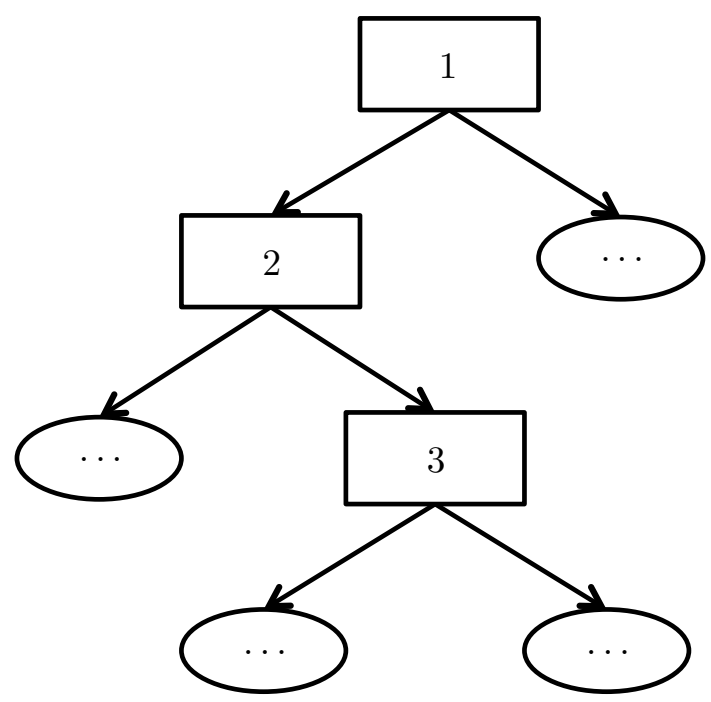

Fig. 3. Fragment of a FOLDT with a complex left-ancestor relation.

ancestors. The union of the local and global predecessors is called the set of predecessors of $\xi$. The positive predecessors of $\xi$ are the positive literals in the set of predecessors of the literal. A variable in an occurrence of a negative literal $\xi$ is covered if it occurs in a positive predecessor of $\xi$, otherwise it is open. Variables in an occurrence of a positive literal are always covered. The basic building blocks of the node labelings are relation symbols, which are inherently safe.

Example 3. In the FOLDT in Fig. 4 variable $y$ in node 2 is covered by the occurrence of $x$ in $R(x, y)$ in node 1 . The variable $x$ in $\neg T(x, y)$, in node 3 , is covered locally by $S(x)$ in this same conjunction. On the other hand, the variable $y$ is not covered in node 3 , because the root is not a leftish ancestor of node 3 and $y$ does not occur locally.

The definition of a FOLDT allows both covered and open variables. A FOLDT $T$ is safe, if all occurrences of variables are covered. It is readily verified that our definition of the semantics of FOLDTs conforms to the original definition given by Blockeel and De Raedt [1, Fig. 2], at least when the FOLDT is safe.

\section{The expressive power of safe FOLDTs}

Our main result characterizes the expressive power of FOLDTs as follows.

Theorem 1. A Boolean query is expressible by a safe FOLDT if and only if it is expressible by a boolean combination of safe existential FOL sentences. 


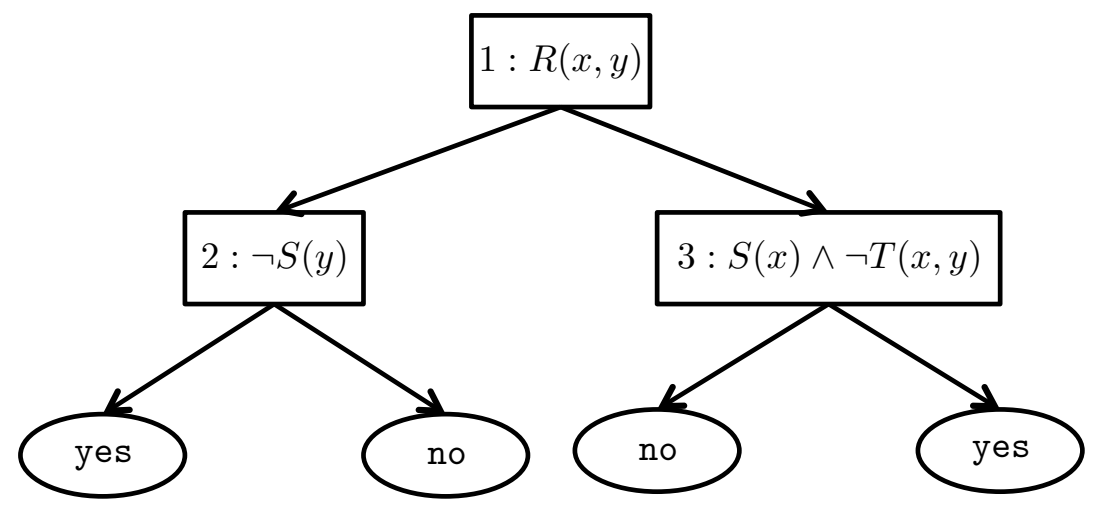

Fig. 4. The occurrences of $y$ in nodes 2 and 3 are not covered.

Here, an existential FOL formula is of the form $(\exists \bar{y}) \psi(\bar{x}, \bar{y})$, where $\psi$ is quantifier-free and $\bar{x}$ and $\bar{y}$ are sets of variables. In other words, a formula is existential if it can be written such that all the quantifiers are in front (prenex normal form) and are existential. An existential FOL sentence is an existential FOL formula without free variables. Boolean combinations are then built up from existential sentences using conjunction, disjunction, and negation. The existential fragment of FOL is usually denoted by $\Sigma_{1}$ [4]. We denote the class of boolean combinations of $\Sigma_{1}$-sentences by $B C\left(\Sigma_{1}\right)$. The class $B C\left(\Sigma_{1}\right)$ is thus a subset of the class of boolean combinations of $\Sigma_{1}$-formulas.

The notion of safety for $B C\left(\Sigma_{1}\right)$-sentences is defined in the following standard way [10]. Let $\chi$ be a $B C\left(\Sigma_{1}\right)$-sentence. For $\chi$ to be safe every $\Sigma_{1}$-sentence in $\chi$ must be safe, defined as follows. Let $\varphi \equiv(\exists \bar{x}) \psi(\bar{x})$ be an existential sentence where $\psi$ is quantifier-free. For $\varphi$ to be safe, we require $\psi$ to be in DNF, and furthermore we require that in every disjunct of $\psi$, every variable is "covered". Here, a variable is covered if it occurs in a positive literal.

The reader should not be lulled into interpreting our theorem as merely stating that the FOL translation $\Phi_{T}$ of a FOLDT $T$ is in $B C\left(\Sigma_{1}\right)$; in fact, it is not. Indeed, the gist of the proof of the only-if direction, shown in Lemma 1, consists of showing that $\Phi_{T}$, for any safe FOLDT, can always be simplified into an equivalent $B C\left(\Sigma_{1}\right)$-sentence. Example 1 already gave an example of this simplification. The if direction of the theorem is proven by Lemmas 2, 3, 4, 5 and 6 .

Lemma 1. Let $T$ be a safe FOLDT. Then $\Phi_{T}$ can be equivalently expressed as a boolean combination of safe $\Sigma_{1}$-sentences.

Proof. Firstly, we show the conversion of $\Phi_{T}$ into a $B C\left(\Sigma_{1}\right)$ sentence. Secondly, it is proven that if $T$ is safe, then $\Phi_{T}$ is also safe. 
$\Phi_{T}$ can be equivalently expressed as a $B C\left(\Sigma_{1}\right)$ sentence: First, we prove that for any node $n$ in $T, \alpha_{n}$ can be written in the form:

$$
\alpha_{n}(\bar{x}) \equiv \psi_{n}(\bar{x}) \wedge \phi_{n}
$$

where $\psi_{n}$ is a conjunction of literals (in particular, $\psi_{n}$ is quantifier-free) and $\phi_{n}$ is a conjunction of negated $\Sigma_{1}$-sentences (in particular, $\phi_{n}$ is in $B C\left(\Sigma_{1}\right)$ ). We prove this by induction on the position of $n$ in $T$.

Base case: $n$ is the root of $T$ Trivial, because $\alpha_{n} \equiv$ true, so we can choose true both for $\psi_{n}$ and $\phi_{n}$.

Induction: $n$ is a descendant of the root Let $p$ be the parent node of $n$. The induction hypothesis states that $\alpha_{p}(\bar{x}) \equiv \psi_{p}(\bar{x}) \wedge \phi_{p}$. Node $n$ is either the left or the right child of $p$. Let $\bar{y}$ be the variables in $\lambda_{p}$ that do not yet belong to $\bar{x}$. Let $\bar{x}^{\prime}=\bar{x} \cup \bar{y}$.

If $n$ is the left child of $p$, we have:

$$
\begin{aligned}
\alpha_{n}\left(\bar{x}^{\prime}\right) & \equiv \alpha_{p}(\bar{x}) \wedge \lambda_{p}(\bar{x}, \bar{y}) \\
& \equiv\left[\lambda_{p}(\bar{x}, \bar{y}) \wedge \psi_{p}(\bar{x})\right] \wedge \phi_{p}
\end{aligned}
$$

So we can choose $\psi_{n}\left(\bar{x}^{\prime}\right) \equiv \lambda_{p}(\bar{x}, \bar{y}) \wedge \psi_{p}(\bar{x})$ and $\phi_{n} \equiv \phi_{p}$.

If $n$ is the right child of $p$, we have:

$$
\begin{aligned}
\alpha_{n}(\bar{x}) & \equiv \alpha_{p}(\bar{x}) \wedge \neg(\exists \bar{x}, \bar{y})\left[\alpha_{p}(\bar{x}) \wedge \lambda_{p}(\bar{x}, \bar{y})\right] \\
& \equiv \psi_{p}(\bar{x}) \wedge \phi_{p} \wedge \neg(\exists \bar{x}, \bar{y})\left[\psi_{p}(\bar{x}) \wedge \phi_{p} \wedge \lambda_{p}(\bar{x}, \bar{y})\right] \\
& \equiv \psi_{p}(\bar{x}) \wedge\left(\phi_{p} \wedge\left(\neg \phi_{p} \vee \neg(\exists \bar{x}, \bar{y})\left[\psi_{p}(\bar{x}) \wedge \lambda_{p}(\bar{x}, \bar{y})\right]\right)\right) \\
& \equiv \psi_{p}(\bar{x}) \wedge\left(\phi_{p} \wedge \neg(\exists \bar{x}, \bar{y})\left[\psi_{p}(\bar{x}) \wedge \lambda_{p}(\bar{x}, \bar{y})\right]\right)
\end{aligned}
$$

So we can choose $\psi_{n}(\bar{x}) \equiv \psi_{p}(\bar{x})$ and $\phi_{n} \equiv \phi_{p} \wedge \neg(\exists \bar{x}, \bar{y})\left[\psi_{p}(\bar{x}) \wedge \lambda_{p}(\bar{x}, \bar{y})\right]$, because both $\psi_{p}$ and $\lambda_{p}$ are quantifier-free.

Secondly, the $\beta$ formula of any node $n$ in FOLDT $T$ can now be converted into a $B C\left(\Sigma_{1}\right)$ formula as follows:

$$
\begin{aligned}
\beta_{n} & \equiv(\exists \bar{x}) \alpha_{n}(\bar{x}) \\
& \equiv\left((\exists \bar{x}) \psi_{n}(\bar{x})\right) \wedge \phi_{n}
\end{aligned}
$$

Finally, we know that $\Phi_{T}$ is the disjunction of the $\beta$ sentences of the yeslabeled leaves. Thus $\Phi_{T}$ is a $B C\left(\Sigma_{1}\right)$ sentence; note that $\Phi_{T}$ is in DNF.

$T$ is safe, then $\Phi_{T}$ is safe: If $T$ is safe, each occurrence of a variable if covered, either locally or by a leftish ancestor. Note in the recursive definition of the $\alpha$ formula, that the conjunctions of all leftish ancestors of a node are combined into a single conjunction, thus constructing a safe existential sentence.

The if-direction of the theorem follows from three basic constructions: 
1. The conjunction of two safe FOLDT-expressible queries is safe FOLDTexpressible. Indeed, if we have two safe FOLDTs $T_{1}$ and $T_{2}$ then we can form their conjunction by attaching a copy of $T_{2}$ at every leaf node of $T_{1}$ labeled yes. This construction is only correct if we make sure in advance (without loss of generality) that $T_{1}$ and $T_{2}$ have disjoint sets of non-output variables. The output variables of the resulting FOLDT are the union of those of $T_{1}$ and $T_{2}$.

2. The negation of a safe FOLDT-expressible query is safe FOLDT-expressible. Indeed, to negate a safe FOLDT it suffices to swap the leaf labels yes and no.

3. Every safe $\Sigma_{1}$-expressible query is expressible by a safe FOLDT. Indeed, consider a safe $\Sigma_{1}$-sentence $\sigma$ of the form $(\exists \bar{x}) \psi(\bar{x})$. We have $\psi$ in DNF: $\gamma_{1} \vee \cdots \vee \gamma_{\ell}$. We construct a FOLDT for $\sigma$ as depicted in Fig. 5. The root is labeled $\gamma_{1}$. From the root descends a chain of right children, labeled $\gamma_{2}$ until $\gamma_{\ell}$. Every node on this linear chain, including the root, gets as left child a leaf labeled yes. Finally, the rightmost node on the chain (the one labeled with $\gamma_{\ell}$ ) gets as right child a leaf labeled no.

We prove in Lemmas 4, 5 and 6 that the above constructions are correct. First we prove two auxiliary lemmas.

Lemma 2. Let $T$ be a FOLDT, let $p$ be a node in $T$ and let $n$ and $m$ be the respective left- and right child of $p$. Then:

$$
\beta_{n} \vee \beta_{m} \equiv \beta_{p}
$$

Proof.

$$
\begin{aligned}
& \beta_{n} \vee \beta_{m} \\
& \left.\equiv \quad(\exists \bar{x}, \bar{y})\left(\alpha_{p}(\bar{x}) \wedge \lambda_{p}(\bar{x}, \bar{y})\right)\right) \vee \\
& \left((\exists \bar{x}) \alpha_{p}(\bar{x}) \wedge \neg(\exists \bar{x}, \bar{y})\left(\alpha_{p}(\bar{x}) \wedge \lambda_{p}(\bar{x}, \bar{y})\right)\right) \\
& \equiv \quad\left((\exists \bar{x}) \alpha_{p}(\bar{x}) \vee(\exists \bar{x}, \bar{y})\left(\alpha_{p}(\bar{x}) \wedge \lambda_{p}(\bar{x}, \bar{y})\right)\right) \wedge \\
& \left((\exists \bar{x}, \bar{y})\left(\alpha_{p}(\bar{x}) \wedge \lambda_{p}(\bar{x}, \bar{y})\right) \vee \neg(\exists \bar{x}, \bar{y})\left(\alpha_{p}(\bar{x}) \wedge \lambda_{p}(\bar{x}, \bar{y})\right)\right) \\
& \equiv \quad(\exists \bar{x}) \alpha_{p}(\bar{x})
\end{aligned}
$$

Here, $\bar{y}$ is the set of free variables in $\lambda_{p}$. We take the following steps in formula:

1. Replacing the $\beta$-formulas by their definition.

2. Distributing the first disjunct over the two parts of the second disjunct.

3. In the first conjunct of 2 , the second part is a specialization of the first part, thus we may drop the second part. In the second conjunct, a tautology has been created. 
Lemma 3. Let $T$ be a FOLDT, let $p$ be a node in $T$ and let $n$ and $m$ be the respective left- and right child of $p$. Then:

$$
\beta_{n} \wedge \beta_{m} \equiv \text { false }
$$

Proof. Let $\bar{x}$ be the set of free variables in $\alpha_{p}$ and let $\bar{y}$ be the free variables in $\lambda_{p}$ that are not in $\bar{x}$.

$$
\begin{array}{cc} 
& \beta_{n} \wedge \beta_{m} \\
\equiv \quad & (\exists \bar{x}, \bar{y})\left(\alpha_{p}(\bar{x}) \wedge \lambda_{p}(\bar{x}, \bar{y})\right) \wedge \\
\equiv \quad & \quad \text { false }
\end{array}
$$

Lemma 4. Let $T_{1}$ and $T_{2}$ be two safe FOLDTs. Suppose, without loss of generality, that the sets of variables in $T_{1}$ and $T_{2}$ are disjoint. The conjunction construction is safe and correct.

Proof. Let $T$ be the FOLDT constructed to express the conjunction of FOLDTs $T_{1}$ and $T_{2}$.

If both $T_{1}$ and $T_{2}$ are safe, then so is $T$. Let $n$ be an internal node of $T$. It is easily verified that all occurrences of variables in the conjunction labeling $n$, $\lambda_{n}$, are covered. If an occurrence of a variable is not covered, it would also be "uncovered" in $T_{1}$ or $T_{2}$, because the structure and labeling are preserved.

To prove the correctness, we need to show that:

$$
\begin{aligned}
\bigvee_{\text {yes-leaf } \ell \text { in T }} \beta_{\ell} & \equiv \Phi_{T_{1}} \wedge \Phi_{T_{2}} \\
& \equiv \bigvee_{\text {yes-leaf } \ell_{1} \text { in } \mathrm{T}_{1}} \beta_{\ell_{1}}^{T_{1}} \wedge \bigvee_{\text {yes-leaf } \ell_{2} \text { in } \mathrm{T}_{2}} \beta_{\ell_{2}}^{T_{2}} \\
& \equiv{ }_{\text {yes-leaf } \ell_{1} \text { in } \mathrm{T}_{1}, \ell_{2} \text { in } \mathrm{T}_{2}} \beta_{\ell_{1}}^{T_{1}} \wedge \beta_{\ell_{2}}^{T_{2}}
\end{aligned}
$$

Let $\ell$ be a yes-leaf of $T$. Leaf $\ell$ corresponds to a yes-leaf $\ell_{2}$ from a copy of $T_{2}$. The copy of $T_{2}$ replaces a yes-leaf $\ell_{1}$ from $T_{1}$. Thus we denote $\ell$ as the couple $\left(\ell_{1}, \ell_{2}\right)$. We have that $\beta_{\ell_{1}}^{T_{1}} \equiv\left(\exists \bar{y}_{1}\right) \alpha_{\ell_{1}}^{T_{1}}\left(\bar{x}_{1}, \bar{y}_{1}\right)$, where $\bar{y}_{1}$ is the set of free variables in $\alpha_{\ell_{1}}^{T_{1}}$. We need to prove that

$$
\beta_{\ell}^{T} \equiv \beta_{\ell_{1}}^{T_{1}} \wedge \beta_{\ell_{2}}^{T_{2}} .
$$

To this end, we show that the $\alpha$-formula of each node $n$, equal to $\ell_{1}$ or a descendent of $\ell_{1}$, can be converted to the form:

$$
\alpha_{n}(\bar{x}) \equiv \alpha_{\ell_{1}}^{T_{1}}\left(\bar{x}_{1}\right) \wedge\left(\psi_{n}\left(\bar{x}_{2}\right) \wedge \phi_{n}\right)
$$


Here, $\bar{x}_{1}$ is the set of free variables in $\alpha_{\ell_{1}}^{T_{1}}, \bar{x}_{2}$ is the set of free variables in $\psi_{n}$, $\bar{x}$ is the union of $\bar{x}_{1}$ and $\bar{x}_{2}, \psi_{n}$ is a conjunction of literals (in particular, $\psi_{n}$ is quantifier-free), $\phi_{n}$ is a conjunction of negated $\Sigma_{1}$-formulas (in particular, $\phi_{n}$ is in $\left.B C\left(\Sigma_{1}\right)\right)$ and $\psi_{n} \wedge \phi_{n} \equiv \alpha_{\ell_{2}}^{T_{2}}$. This last property can be verified in the same way as in the proof of Lemma 1 .

If $n=\ell_{1}$, the claim holds trivially, as we can choose $\psi_{n} \equiv$ true $\equiv \phi_{n}$.

If $n \neq \ell_{1}$, let $p$ be the parent node of $n$, by the induction hypothesis we know:

$$
\alpha_{p}(\bar{x}) \equiv \alpha_{\ell_{1}}^{T_{1}}\left(\bar{x}_{1}\right) \wedge\left(\psi_{n}\left(\bar{x}_{2}\right) \wedge \phi_{n}\right)
$$

There are two cases:

1. Node $n$ is the left child of $p$ : Let $\bar{y}$ be the set of free variables in $\lambda_{p}$ that are not in $\bar{x}_{2}$

$$
\begin{aligned}
\alpha_{n}(\bar{x}) & \equiv \alpha_{p}\left(\bar{x}^{\prime}\right) \wedge \lambda_{p}\left(\bar{x}_{2}, \bar{y}\right) \\
& \equiv \alpha_{\ell_{1}}^{T_{1}}\left(\bar{x}_{1}\right) \wedge\left(\left(\psi_{p}\left(\bar{x}_{2}\right) \wedge \lambda_{p}\left(\bar{x}_{2}, \bar{y}\right)\right) \wedge \phi_{p}\right)
\end{aligned}
$$

So we can choose $\psi_{n}\left(\bar{x}_{2}^{\prime}\right) \equiv \psi_{p}\left(\bar{x}_{2}\right) \wedge \lambda_{p}\left(\bar{x}_{2}, \bar{y}\right)$ with $\bar{x}_{2}^{\prime}=\bar{x}_{2} \cup \bar{y}$ and $\phi_{n} \equiv \phi_{p}$. 2. Node $n$ is the right child of $p$ :

$$
\begin{aligned}
\alpha_{n}(\bar{x}) \equiv & \alpha_{p}\left(\bar{x}^{\prime}\right) \wedge \neg\left(\exists \bar{x}^{\prime}, \bar{y}\right)\left[\alpha_{p}(\bar{x}) \wedge \lambda_{p}\left(\bar{x}_{2}, \bar{y}\right)\right] \\
\equiv & \alpha_{\ell_{1}}^{T_{1}}\left(\bar{x}_{1}\right) \wedge \psi_{p}\left(\bar{x}_{2}\right) \wedge \phi_{p} \wedge \\
& \neg\left(\left(\exists \bar{x}_{1}\right)\left[\alpha_{\ell_{1}}^{T_{1}}\left(\bar{x}_{1}\right)\right] \wedge\left(\exists \bar{x}_{2}, \bar{y}\right)\left[\psi_{p}\left(\bar{x}_{2}\right) \wedge \lambda_{p}\left(\bar{x}_{2}, \bar{y}\right)\right] \wedge \phi_{p}\right) \\
\equiv & \alpha_{\ell_{1}}^{T_{1}}\left(\bar{x}_{1}\right) \wedge \psi_{p}\left(\bar{x}_{2}\right) \wedge \phi_{p} \wedge \\
& \left(\neg \phi_{p} \vee \neg\left(\exists \bar{x}_{1}\right) \alpha_{\ell_{1}}^{T_{1}}\left(\bar{x}_{1}\right) \vee \neg\left(\exists \bar{x}_{2}, \bar{y}\right)\left[\psi_{p}\left(\bar{x}_{2}\right) \wedge \lambda_{p}\left(\bar{x}_{2}, \bar{y}\right)\right]\right) \\
\equiv & \alpha_{\ell_{1}}^{T_{1}}\left(\bar{x}_{1}\right) \wedge\left(\psi_{p}\left(\bar{x}_{2}\right) \wedge \phi_{p}\right) \wedge \\
& \left(\neg\left(\exists \bar{x}_{1}\right) \alpha_{\ell_{1}}^{T_{1}}\left(\bar{x}_{1}\right) \vee \neg\left(\exists \bar{x}_{2}, \bar{y}\right)\left[\psi_{p}\left(\bar{x}_{2}\right) \wedge \lambda_{p}\left(\bar{x}_{2}, \bar{y}\right)\right]\right)
\end{aligned}
$$

In formula 4 we encounter an interesting situation. If $\neg\left(\exists \bar{x}_{1}\right) \alpha_{\ell_{1}}^{T_{1}}\left(\bar{x}_{1}\right)$ evaluates to true, the first conjunct $\left(\alpha_{\ell_{1}}^{T_{1}}\left(\bar{x}_{1}\right)\right)$ will never evaluate to true and thereby the formula will evaluate to false. However, if $\neg\left(\exists \bar{x}_{1}\right) \alpha_{\ell_{1}}^{T_{1}}\left(\bar{x}_{1}\right)$ would evaluate to true, we could not have arrived at $\ell_{1}$. Obviously, as we are now in a descendant of $\ell_{1}$, this is a contradiction. The subformula can thus be dropped from the disjunction, so we can choose:

$$
\begin{aligned}
\psi_{n}\left(\bar{x}_{2}\right) & \equiv \psi_{p}\left(\bar{x}_{2}\right) \\
\phi_{n} & \equiv \phi_{p} \wedge \neg\left(\exists \bar{x}_{2}, \bar{y}\right)\left[\psi_{p}\left(\bar{x}_{2}\right) \wedge \lambda_{p}\left(\bar{x}_{2}, \bar{y}\right)\right]
\end{aligned}
$$

Hereby the statement is proven correct.

Lemma 5. Let $T$ be a safe FOLDT. The negation construction is safe and correct. 
Proof. A FOLDT is safe if all occurrences of variables are covered. The difference between $T$ and $T_{\neg}$ are the leafs labels. The leafs are not labeled with conjunctions of literals and the structure is not changed. Therefore, $T_{\neg}$ is also safe.

Lemmas 2 and 3 state that if a certain node evaluates to true, exactly one one leaf descending from that node also evaluates to true. By definition, we know that $\beta_{r} \equiv$ true, where $r$ is the root node of $T$. As a result, for any interpretation, exactly one leaf evaluates to true whereas all others will evaluate to false. Clearly, by inverting the leaf labels, all interpretations accepted by $T$ are rejected by $T_{\neg}$ and vice versa.

Lemma 6. Let $T_{\sigma}$ be the tree constructed as above to express the safe $\Sigma_{1}$ sentence $\sigma \equiv(\exists \bar{x})\left(\gamma_{1}\left(\bar{x}_{1}\right) \vee \ldots \vee \gamma_{\ell}\left(\bar{x}_{\ell}\right)\right.$. Then $T_{\sigma}$ is a safe FOLDT and $\Phi_{T_{\sigma}} \equiv \sigma$.

Proof. Because $\sigma$ is a safe existential sentence and each conjunct labels one node, each variable is locally covered.

We prove the equivalence by induction on $\ell$.

$\ell=1$ :

$$
\begin{aligned}
\Phi_{T_{\sigma}} & \equiv\left(\exists \bar{x}_{1}\right) \gamma_{1}\left(\bar{x}_{1}\right) \\
& \equiv \sigma
\end{aligned}
$$

$\ell>1$ : Let $\sigma^{\prime}$ be $\sigma$ without the last disjunct. Then by the induction hypothesis we know that:

$$
\sigma^{\prime} \equiv(\exists \bar{x})\left[\gamma_{1}\left(\bar{x}_{1}\right) \vee \ldots \vee \gamma_{\ell-1}\left(\bar{x}_{\ell-1}\right)\right] \equiv \Phi_{T_{\sigma^{\prime}}}
$$

By construction, $T_{\sigma}$ is obtained from $T_{\sigma^{\prime}}$ by replacing the no-labeled leaf by an internal node $m$ labeled with $\gamma_{\ell}\left(\bar{x}_{\ell}\right)$, and with the left child labeled yes and the right child labeled no. From Lemmas 2 and 3 it readily follows that the $\alpha$-formula of node $m$ is equivalent to $\neg \Phi_{T_{\sigma^{\prime}}}$. Hence, the sentence describing the semantics of $T_{\sigma}$ is:

$$
\begin{aligned}
\Phi_{T_{\sigma}} & \equiv \Phi_{T_{\sigma^{\prime}}} \vee\left[\neg \Phi_{T_{\sigma^{\prime}}} \wedge \gamma_{\ell}\left(\bar{x}_{\ell}\right)\right] \\
& \equiv \Phi_{T_{\sigma^{\prime}}} \vee \gamma_{\ell}\left(\bar{x}_{\ell}\right) \\
& \equiv \sigma
\end{aligned}
$$

\section{Discussion}

Our result places the expressive power of FOLDTs at a rather low position in the quantifier alternation hierarchy for first-order logic [4]. The safe existential sentences are a subset of $\Sigma_{1}$, the existential fragment of first-order logic. The next level in this hierarchy is $\Sigma_{2}$, consisting of all formulas that can be put in prenex form with a quantifier prefix of the form $\exists^{*} \forall^{*}$. Similarly, $\Pi_{2}$ consists of 


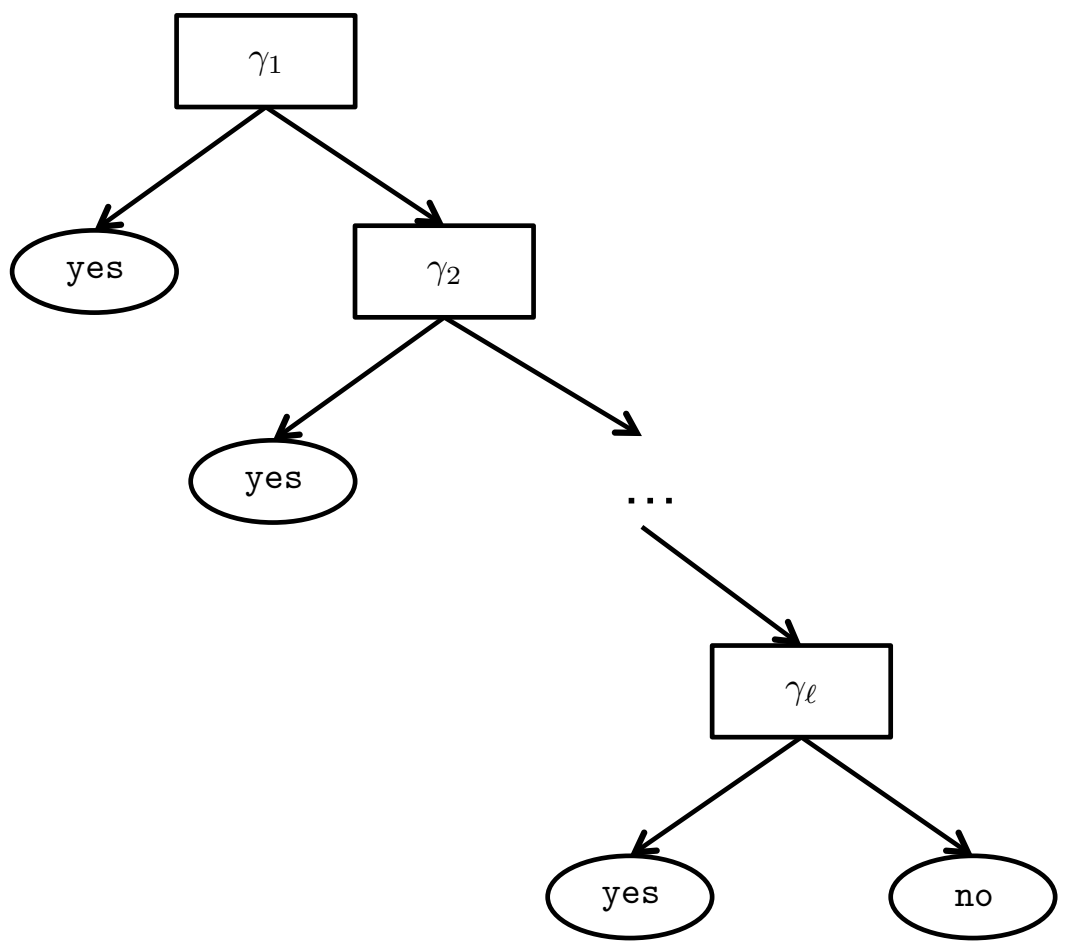

Fig. 5. Illustration of a FOLDT expressing a $\Sigma_{1}$-formula.

the $\forall^{*} \exists^{*}$ formulas. It is easy to see that $B C\left(\Sigma_{1}\right)$ formulas can be put both in $\Sigma_{2}$ form and in $\Pi_{2}$ form. This places the FOLDT-expressible queries in the class known as $\Delta_{2}$ : the queries expressible both by a $\Sigma_{2}$-formula and by a $\Pi_{2}$-formula.

Now it is known that there are queries expressible in $\Pi_{2}$ but not in $\Sigma_{2}$, and vice versa, even in restriction to finite interpretations [4]. Any such queries are not FOLDT-expressible. For example, the boolean query mentioned in the Introduction "there exists a blue node with edges to all red nodes", $(\exists x)(\forall y)(B(x) \wedge$ $(R(y) \rightarrow E(x, y)))$, is a typical example of a query expressible in $\Sigma_{2}$ but not in $\Pi_{2}$, and, consequently, not as a FOLDT. Likewise, the boolean query "all blue nodes have an edge to some red node", $(\forall x)(\exists y)(B(x) \rightarrow(R(y) \wedge E(x, y)))$ is in $\Pi_{2}$ but not in $\Sigma_{2}$ and hence again not FOLDT-expressible.

In the present work we have focused on safe FOLDTs and the safe fragment of existential formulas. This is due to the nature of Prolog evaluation. In future work, we would like to dig deeper into the "full" semantics of FOLDTs, i.e., we would like to incorporate unsafe FOLDTs in our definition of the semantics.

Most commonly, ILP learners induce clausal theories, i.e., conjunctions of universally quantified clauses (disjunctions of literals). Since a univerally quantified disjunction of literals amounts to a negated $\Sigma_{1}$-formula, clausal theories 
thus correspond to the fragment of $B C\left(\Sigma_{1}\right)$ formed by all conjunctions of negated $\Sigma_{1}$-formulas. (A safety notion appropriate for clauses an be analogously defined.)

\section{Acknowledgment}

We thank Hendrik Blockeel and Jan Struyf for interesting discussions and help with the Tilde system. We also thank the anonymous referees for their helpful comments on an extended abstract of this paper.

\section{References}

1. Blockeel, H., De Raedt, L.: Top-down induction of first-order logical decision trees. Artificial Intelligence 101, 285-297 (1998)

2. Blockeel, H., Dehaspe, L., Ramon, J., Struyf, J., Van Assche, A., Vens, C., Fierens, D.: The ace data mining system (2008)

3. Blockeel, H., Dehaspe, L., Demoen, B., Janssens, G., Ramon, J., Vandecasteele, H.: Improving the efficiency of inductive logic programming through the use of query packs. Journal of Artificial Intelligence Research 16, 135-166 (2002), https://lirias.kuleuven.be/handle/123456789/123799

4. Chandra, A., Harel, D.: Structure and complexity of relational queries. Journal of Computer and System Sciences 25(1), 99-128 (1982)

5. De Raedt, L.: Logical and Relational Learning. Cognitive Technologies, Springer (2008)

6. Martin, E., Sharma, A., Stephan, F.: A general theory of deduction, induction, and learning. In: Proceedings of the 4th International Conference on Discovery Science. pp. 228-242. DS '01, Springer-Verlag, London, UK (2001)

7. Ohsherson, D., Weinstein, S.: Identification in the limit of first order structures. Journal of Philiosophical Logic 15, 55-81 (1986)

8. Osherson, D., Stob, M., Weinstein, S.: A universal inductive inference machine. The Journal of Symbolic Logic 56, 661-672 (1991)

9. Terwijn, S.: Learning and computing in the limit. In: Logic Colloquium '02, Lect. Notes Log., vol. 27, pp. 349-359. Assoc. Symbol. Logic, La Jolla, CA (2006)

10. Ullman, J.: Principles of Database and Knowledge-Base Systems, vol. I. Computer Science Press (1988) 